\title{
Fast axial and lateral displacement estimation in myocardial elastography based on RF signals with predictions
}

\author{
Yaonan Zhang*, Tingting Sun, Yueyang Teng, Hong Li and Yan Kang \\ Sino-Dutch Biomedical and Information Engineering School, Northeastern University, Shenyang, \\ China
}

\begin{abstract}
Myocardial elastography (ME) is a strain imaging technique used to diagnose myocardial diseases. Axial and lateral displacement calculations are pre-conditions of strain image acquisition in ME. W.N. Lee et al. proposed a normalized cross-correlation (NCC) and recorrelation method to obtain both axial and lateral displacements in ME. However, this method is not noise-resistant and of high computational cost. This paper proposes a predicted fast NCC algorithm based on W.N. Lee's method, with the additions of sum-table NCC and a displacement prediction algorithm, to obtain efficient and accurate axial and lateral displacements. Compared to experiments based on the NCC and recorrelation methods, the results indicate that the proposed NCC method is much faster (predicted fast NCC method, $69.75 \mathrm{~s}$ for a $520 \times 260$ image; NCC and recorrelation method, $1092.25 \mathrm{~s}$ for a $520 \times 260$ image) and demonstrates better performance in eliminating decorrelation noise (SNR of the axial and lateral strain using the proposed method, 5.87 and 1.25, respectively; SNR of the axial and lateral strain using the NCC and recorrelation method, 1.48 and 1.09 , respectively).
\end{abstract}

Keywords: Myocardial elastography (ME), displacement estimation, normalized cross-correlation, recorrelation, prediction

\section{Introduction}

Myocardial elastography (ME) is applied to diagnose cardiac disease by determining myocardial deformations. Although tagged magnetic resonance imaging (tMRI) [1] is regarded as the premier method of noninvasive cardiac disease diagnosis, ME is still widely used due to its low cost, higher temporal resolution and portability.

ME was first proposed by E.E. Konofagou, et al. [2, 3], and functions as a strain imaging technique based on ultrasound to assess myocardial function. Conventional elastography depends on external stimulus, while ME relies on the intrinsic deformation of the heart which undergoes 3-D movement. Thus, a single calculation of axial deformation is not sufficient for diagnosis of myocardial deformation.

E.E. Konofagou, et al. [2, 3] used a normalized cross-correlation (NCC) method to calculate axial displacement and strain. This was improved by W.N. Lee, et al. [4] by using NCC to extend the

\footnotetext{
*Address for correspondence: Yaonan Zhang, Sino-Dutch Biomedical and Information Engineering School, Northeastern University (Hunnan Campus), P.O.Box H004, 500 Zhihui Dajie, Hunnan District, Shenyang, China. Tel.: +86-24-83685261; Fax: +86-24-83681955; E-mail: zhangyn@bmie.neu.edu.cn.
} 
displacement estimation from 1-D to 2-D. Additionally, W.N. Lee, et al. [5] proposed a recorrelation method to reduce the decorrelation noises of axial and lateral displacements obtained by NCC. Although this method demonstrates advantages such as high accuracy, resolution and relative simplicity in implementation in motion estimation [5], it also has high computation cost, which limits its applicability as real-time elastography imaging. Additionally, although recorrelation can reduce signal decorrelation, its performance is not wholly effective.

This paper proposes a predicted fast NCC method to calculate both axial and lateral displacements accurately and efficiently. First, a sum-table NCC [6] method was used to accelerate computation speed, the search range was subsequently reduced by predicting displacements according to its neighbor displacement.

\section{Methodology}

\subsection{NCC and recorrelation in displacement estimation}

The NCC and recorrelation method proposed by W.N. Lee is a two-step estimation algorithm, as shown in Figure 1(a). In the first estimation, a 1-D NCC matching kernel was employed in a 2-D search in order to simultaneously obtain axial and lateral displacement. In the second estimation, a recorrelation method was used to correct the lateral and axial displacement estimation and reduce decorrelation noise.

\subsubsection{NCC displacement estimation (first estimation)}

NCC has long been regarded as the premier method of motion estimation. A windowing method was applied to each of the RF lines of both the precompressed and postcompressed signals. The NCC between the precompressed window and postcompressed window is defined as [7]:
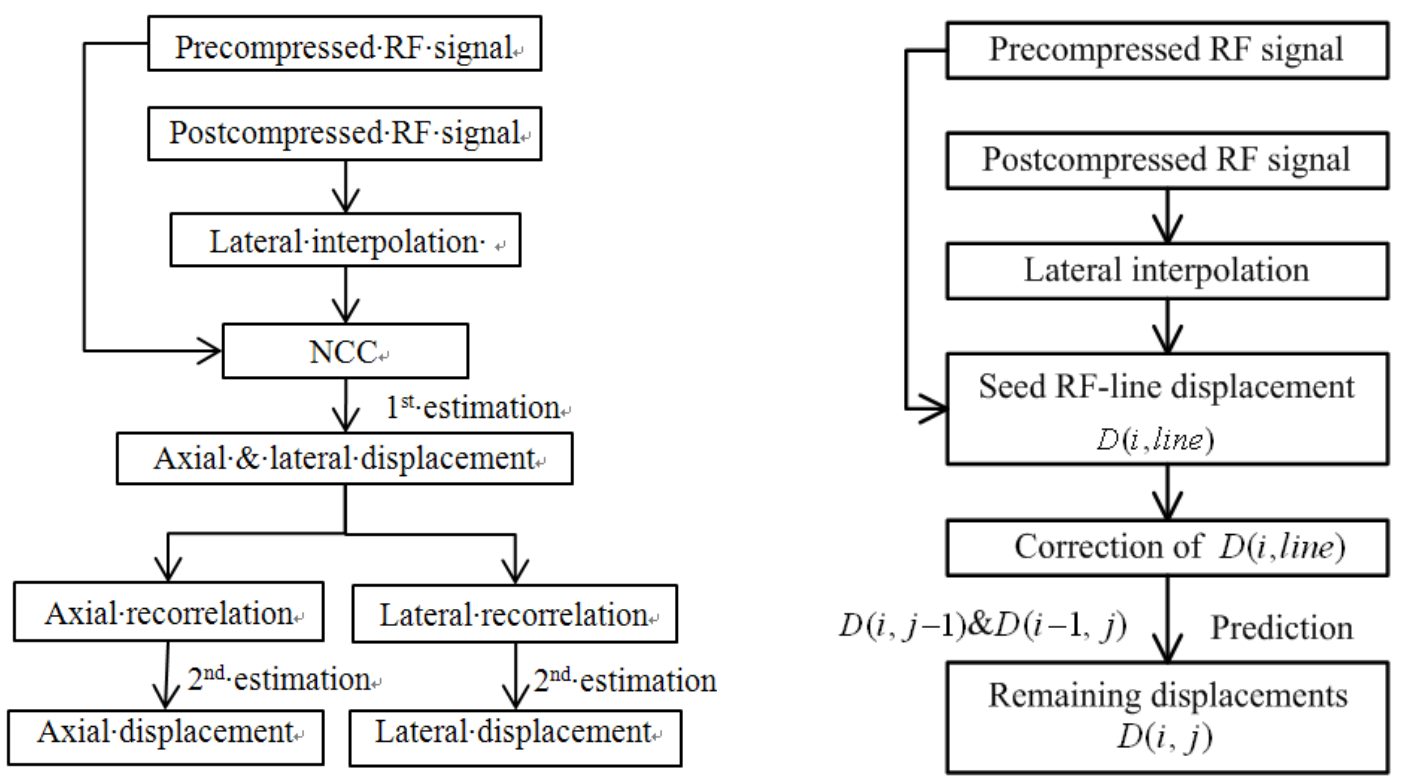

Fig. 1. (a) Diagram of the NCC and recorrelation algorithm; (b) diagram of the predicted fast NCC algorithm, in which D represents both axial and lateral displacement. 


$$
R_{N C C}(u, \tau)=\frac{\sum_{n=u}^{u+W-1} f(n) g(n+\tau)}{\sqrt{\sum_{n=u}^{u+W-1} f^{2}(n) \cdot \sum_{n=u}^{u+W-1} g^{2}(n+\tau)}},\left(\tau_{1} \leq \tau \leq \tau_{2}\right)
$$

where $W$ is the size of window, $u$ is the original position, and $\tau$ is the time shift. The displacement was calculated by determineing the maximum correlation coefficient.

In the first displacement estimation, the NCC method estimating 2-D displacement by a 1-D matching kernel was divided into the three following steps [4]:

1). In order to improve the lateral resolution, a 16:1 linear interpolation was implemented in the lateral direction of the postcompressed RF signal [8];

2). NCC calculation was performed between a segment in the precompressed RF signal and the candidate segment within the axial and lateral searching range of the postcompressed RF signal; the search range consisted of $16([0,15])$ samples in the axial direction and $9( \pm 4)$ RF-lines in the lateral direction. The axial and lateral segment shift which yielded the highest correlation coefficient in the 2-D search range represented the axial and lateral displacement;

3). A three-point interpolation algorithm [8] was performed after the NCC calculation to improve the precision of the cross coefficient peak detection.

\subsubsection{Recorrelation correction of displacement estimation (second estimation)}

The axial (or lateral) signal decorrelation caused by lateral (or axial) movement is the largest source of noise in elastography [9]; a global stretching method [8] has typically been used to correct the signal decorrelation. However, as the prior knowledge of the compressibility is unknown in ME, global stretching is not suitable. The recorrelation method proposed by W.N. Lee to eliminate the signal decorrelation consists of the following two steps:

1). Rearrangement of the postcompressed RF signal by shifting the RF segments according to the first lateral (or axial) displacement obtained by NCC;

2). Calculation of the axial (or lateral) displacement through the NCC method between the precompressed RF signal and the rearranged postcompressed RF signal, rather than using the original postcompressed RF signal.

\subsection{Predicted fast NCC in displacement estimation}

The NCC calculation was improved by a sum-table algorithm to reduce the redundant calculation created by the high window overlap among successive windows. In addition, to further decrease the high computational cost created by the exhaustive search during the NCC calculation of each window, the searching range was reduced by predicting the displacement using the previously calculated value.

\subsubsection{Sum-table NCC}

To improve the computational speed, a fast NCC based on the sum table [6] was used in the predicted fast NCC algorithm, by re-writing the $R_{N C C}$ as follows:

$$
R_{N C C}(u, \tau, W)=\frac{S_{f, g}(u+W-1, \tau)-S_{f, g}(u-1, \tau)}{\sqrt{\left(S_{f}^{2}(u+W-1)-S_{f}^{2}(u-1)\right) \cdot\left(S_{g}^{2}(u+W-1+\tau)-S_{g}^{2}(u-1+\tau)\right)}}
$$


where $S$ represents the sum table.

\subsubsection{Predicted NCC of displacement estimation}

As movement in tissue is continuous, the adjacent displacements of tissues are similar. Thus, in the predicted fast NCC method, the similarity of the successive displacements was considered to reduce the search range. Before calculating the displacement of the present window, a displacement prediction was made based on the previously calculated displacement of its neighbor window. Then, the search range of the present window is reduced to the predicted displacement \pm 2 samples in the axial direction and \pm 2 RF-lines in the lateral direction.

The steps of this algorithm are described as follows, and shown in Figure 1(b). First, a 16:1 linear interpolation was performed in the lateral direction of the postcompressed signal to improve the lateral resolution. Second, the axial and lateral displacements of one seed RF-line were calculated using the sum-table NCC method. As the boundary of an image displays much more noise than the middle, the seed RF-line was preferably chosen in the middle of the image. Third, as the subsequent RF-line displacements are affected by the displacements of the seed RF-line, to obtain accurate displacements of the whole image, the outliers in the axial and lateral displacement of the seed RF-line were determined by applying a threshold to the first-order derivative of the displacements and replaced by the linear interpolation of the neighbor points. Finally, the corrected displacements were propagated to the left and right of the seed RF-line by sum-table NCC, taking the neighbor displacement and the weighted average of $D A(i, j-1)$ and $D A(i-1, j)$, as the predicted estimate. Then, the searching range was replaced by the predicted displacement $\pm 2 . D A(i, j)$ and $D L(i, j)$ represent the axial and lateral displacement in the $j$ th column and ith row, respectively.

\subsection{The axial and lateral strain estimation}

Strain can be calculated using the derivation of the displacement. However, the direct derivative operator amplifies the high frequency noise in displacement. This paper utilized a least squares strain estimator (LSQSE) [10] to calculate the axial and lateral strain. To evaluate the effect of the predicted fast NCC method on strain calculation, the signal-to-noise ratio (SNR) of the stain image was calculated [11].

\section{Results}

The predicted fast NCC algorithm proposed in this paper was compared to the NCC and recorrelation algorithm. The results of the NCC and recorrelation algorithm are shown in Figure 2. As shown in Figures 2(a) and 2(e), the axial and lateral displacements were obtained simultaneously by the first estimation. Axial and lateral displacement maps after applying three-point cosine interpolation to the first axial and lateral displacement are shown in Figures 2(b) and 2(f). Figures 2(c) and 2(g) show the second axial and lateral displacement calculated in the second estimation. Figures 2(a) and 2(e) depict a map of the axial and lateral displacements, but contain large amounts of noises. Comparing Figures 2(a) and 2(e) to Figures 2(b) and 2(f), the latter set demonstrates obvious reduction in noises. However, there is no obvious difference between Figures 2(b) and 2(c). Figures 2(d) and 2(h) display the first and second estimation NCC coefficient maps, which show improvement of the second displacement estimation as compared to the first estimation. 


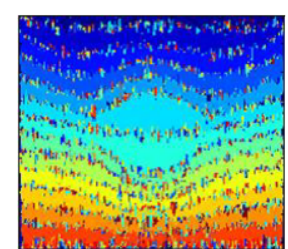

(a)

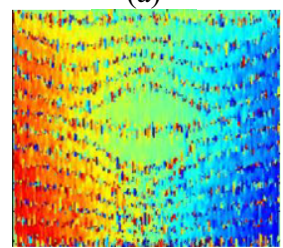

(e)

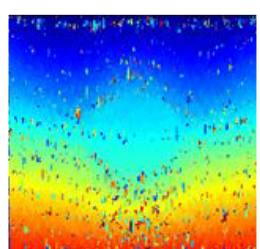

(b)

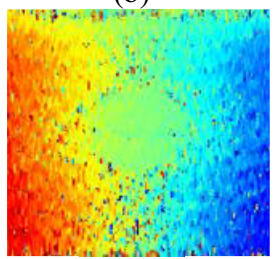

(f)

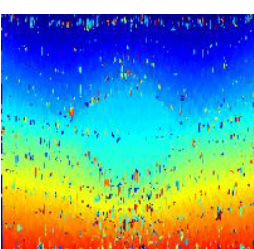

(c)

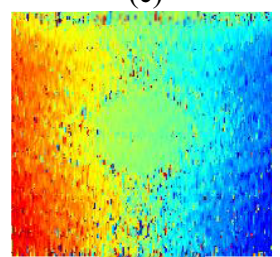

(g)

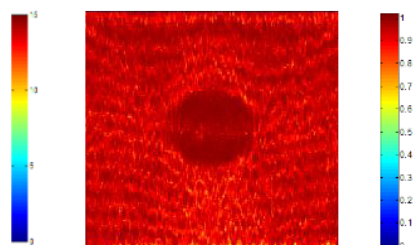

(d)

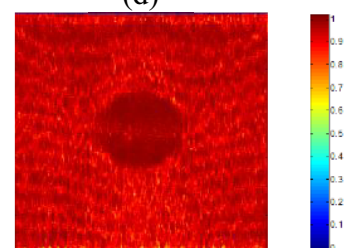

(h)

Fig. 2. NCC and recorrelation algorithm. (a) and (e) show the first axial and lateral displacement estimates; (b) and (f) show the first axial and lateral displacement estimate after cosine interpolation; (c) and (g) show the second axial and lateral displacement; (d) and (h) show the NCC coefficient map of first estimation (without recorrelation) and second estimation (with recorrelation).

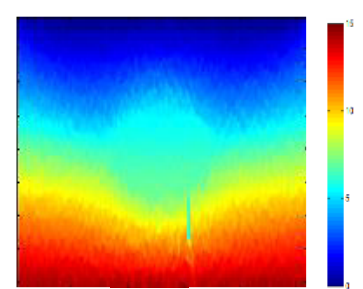

(a)

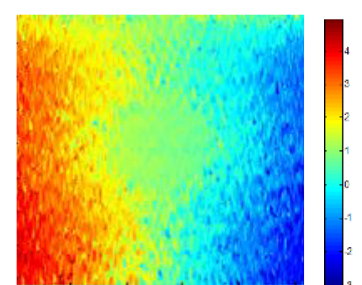

(b)

Fig. 3. Predicted fast NCC algorithm: (a) axial displacement; (b) lateral displacement.

The results of the predicted fast NCC algorithm are shown in Figure 3. Figures 3(a) and 3(b) demonstrate the axial and lateral displacement simultaneously calculated by the predicted fast NCC. Comparing Figures 3(a) and 3(b) to Figures 2(c) and 2(g), it is obvious that better results are obtained by NCC and recorrelation.

For the RF data of $520 \times 260$ pixels used in this paper, the NCC and recorrelation method required took $1092.25 \mathrm{~s}$, while the proposed fast NCC method just required only $69.75 \mathrm{~s}$ on a $2.5 \mathrm{GHz}$ P2 CPU, which is nearly 16 times faster than the NCC and recorrelation method.

Figure 4 displays the strain images obtained from displacements shown in Figures 2(c) and 2(g) and Figures 3(a) and 3(b). The SNRs of Figures 4(a) and 4(b) are 1.48 and 1.09, respectively. The SNRs of

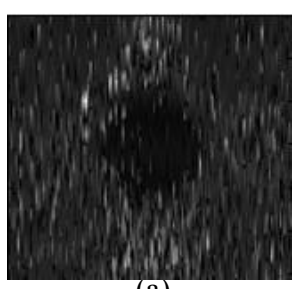

(a)

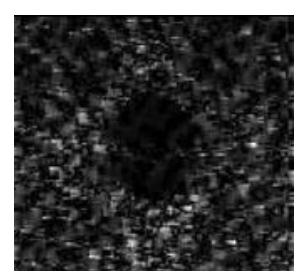

(b)

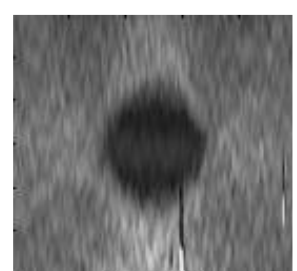

(c)

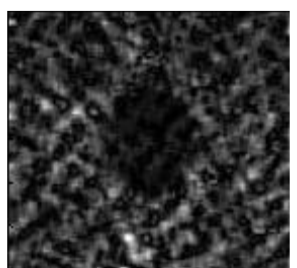

(d)

Fig. 4. Strain images. (a) and (b) show the axial strain and lateral strain obtained from NCC and recorrelation method; (c) and (d) show the axial strain and lateral strain obtained from the predicted fast NCC method. 
Figures 4(c) and 4(d) are 5.86 and 1.25, respectively. Both axial and lateral strain SNR obtained from the predicted fast NCC method showed improvement.

\section{Discussion and conclusion}

In the proposed NCC and recorrelation method, the recorrelation method improved the accuracy of the displacement estimation compared to the first estimation, though no obvious differences were observed between methods after the addition of the three-point cosine interpolation. The correlation of the second displacement estimation also improved (the NCC coefficients of the first and second displacement estimation (mean \pm SD) were $0.9251 \pm 0.0460$ and $0.9583 \pm 0.0309$, respectively).

Although capable of providing a relatively accurate displacement, the recorrelation method demonstrates a high computational cost $(1092.25 \mathrm{~s}$ for a $520 \times 260$ image $)$ and is not noise-resistant. The proposed predicted fast NCC method demonstrates obvious improvement in computational speed (69.75s for a $520 \times 260$ image). These results indicate that the sum-table NCC algorithm doubled the speed of NCC calculation. Another possible reason is that the search range was reduced in each loop from nine lines $([-4,4])$ to five (the predicted lateral displacement \pm 2 ) in the lateral direction and from $16([0,15])$ samples to five (the predicted axial displacement \pm 2$)$ in the axial direction.

By using the predicted fast NCC method, the SNR of both axial and lateral strain were improved from 1.48 to 5.87 and from 1.09 to 1.25 in the axial and lateral directions, respectively. This indicates that the predicted fast NCC method demonstrates better performance in eliminating signal decorrelation than the NCC and recorrelation method.

\section{Acknowledgments}

This work is sponsored by Natural Science Foundation (No. 61201053, 61302013 and 61372014) of China and Natural Science Foundation of Liaoning Province (project 201202071), China.

\section{References}

[1] J. Declerck, T.S. Denney, C. Ozturk, W. O’ Dell and E.R. McVeigh, Left ventricular motion reconstruction from planar tagged MR images: A comparison, Physics in Medicine and Biology 45 (2000), 1611-1632.

[2] E.E. Konofagou, J. D'hooge and J. Ophir, Cardiac elastography-A feasibility study, Ultrasonics Symposium Proceedings 2 (2000), 1273-1276.

[3] E.E. Konofagou, J. D'Hooge and J. Ophir, Myocardial elastography-A feasibility study in vivo, Ultrasound in Medicine \& Biology 28 (2002), 475-482.

[4] W.N. Lee, C.M. Ingrassia and S.D. Fung-Kee-Fung, Theoretical quality assessment of myocardial elastography with in vivo validation, IEEE Transactions on Ultrasonics, Ferroelectrics, and Frequency Control 54 (2007), 2233-2245.

[5] P.M. Embree and W.D. Obrien, Volumetric blood flow via time domain correlation-Experimental verification, IEEE Transactions on Ultrasonics, Ferroelectrics and Frequency Control 37 (1990), 176-189.

[6] J. Luo and E.E. Konofagou, A fast normalized cross-correlation calculation method for motion estimation, IEEE Transactions on Ultrasonics, Ferroelectrics and Frequency Control 57 (2010), 1347-1357.

[7] F. Viola and W.F. Walker, A comparison of the performance of time-delay estimators in medical ultrasound, IEEE Transactions on Ultrasonics, Ferroelectrics and Frequency Control 50 (2003), 392-401.

[8] E.E. Konofagou and J. Ophir, A new elastographic method for estimation and imaging of lateral displacements, lateral strains, corrected axial strains and Poisson's ratios in tissues, Ultrasound in Medicine \& Biology 24 (1998), 1183-1199.

[9] F. Kallel and J. Ophir, Three-dimensional tissue motion and its effect on image noise in elastography, IEEE Transactions on Ultrasonics, Ferroelectrics and Frequency Control 44 (1997), 1286-1296. 
[10] F. Kallel and J. Ophir, A least-squares strain estimator for elastography, Ultrasound Imaging 19 (1997), 195-208.

[11] J. Ophir, S. Alam, B. Garra, F. Kallel, E. Konofagou, T. Krouskop and T. Varghese, Elastography: Ultrasonic estimation and imaging of the elastic properties of tissues, Annual Review of Biomedical Engineering 213 (1999), 203-233. 